\title{
PROPERTIES AND DEVICE APPLICATIONS OF DEEP QUANTUM WELL RESONANT TUNNELING STRUCTURES
}

\author{
I. MEHDI, R.K. MAINS, G.I. HADDAD and U.K. REDDY \\ Center for High-Frequency Microelectronics, Department of Electrical Engineering and Computer Science. The Uninersity of Michigan. \\ Ann Arbor, MI 48109, USA
}

Received 5 June 1989; accepted for publication 20 July 1989

A self-consistent quantum mechanical simulation is used to study the effects of doping. spacer layers, barrier height, barrier width and well doping on the bound state and bound state charge in a deep quantum well resonant tunneling structure. Experimental results for the GaAs and InP heterostructure material system are presented. Finally various device applications, such as microwave video detectors, charge transfer devices, and three terminal devices are proposed.

\section{Introduction}

A resonant tunneling structure (RTS) where the well is made of a smaller bandgap material than the material enclosing the barriers is termed a deep quantum well RTS. By proper selection of the heterostructure material system and device dimensions a RTS with a bound state in the deep quantum well can be obtained. The creation of a bound state, and thus charge in the bound state, gives rise to some interesting properties which are significantly different from those of a conventional RTS.

\section{Theoretical formalism}

A self-consistent simulation has been developed that takes into account the charge in the well. The contact regions outside the double-barrier structure are treated using a Thomas-Fermi equilibrium model, i.e. constant Fermi levels within each region and equilibrium Fermi-Dirac statistics are assumed. Within the double-barrier structure, electron concentrations are obtained from the time-independent Schrödinger equation. The concentrations obtained inside the device are then self-consistently coupled to the Thomas-Fermi model through matching conditions connecting the two contact regions $[1,2]$.

In order to solve for the bound state the energy from the bottom of the well is scanned and a finite difference form of Schrödinger's equation is solved until a well confined wavefunction is obtained. The corresponding wavefunction is normalized to unity and then multiplied by the two dimensional density of states and occupation probability function to obtain the charge density in the well.

It is found that parameters such as barrier height and barrier width though important for peak-to-valley ratio and current density [3] do not affect the charge in the bound state dramatically. Fig. la shows the variation in the position of the bound state and the charge in the bound state as a function of doping in the well and the emitter (emitter and collector dopings are assumed to be the same throughout this paper). Fig. 1b shows the same information for varying spacer layer widths. These figures are for zero bias. The position of the bound state is specified with reference to the band edge outside the barriers which is taken to be zero. For these results the barrier width was $28.2 \mathrm{~A}$. barrier height was $0.25 \mathrm{eV}$, well depth was 0.19 $\mathrm{eV}$, well width was $100 \AA$, electron mass in the emitter and collector was $0.067 m_{1}$, electron mass in the well was $0.056 m_{0}$, electron mass in the 


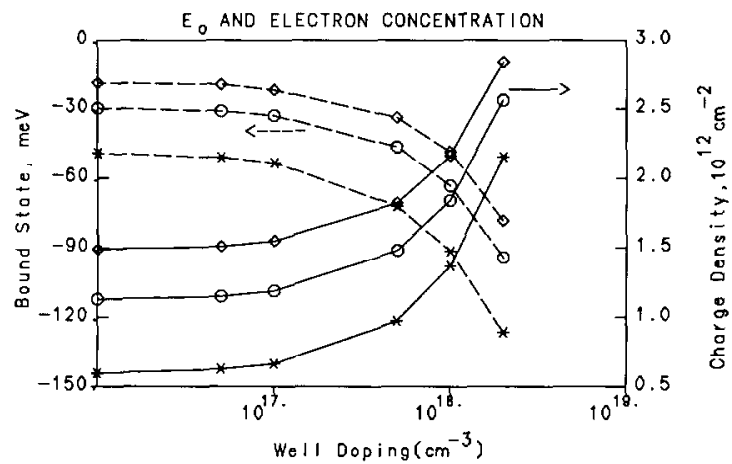

(o)

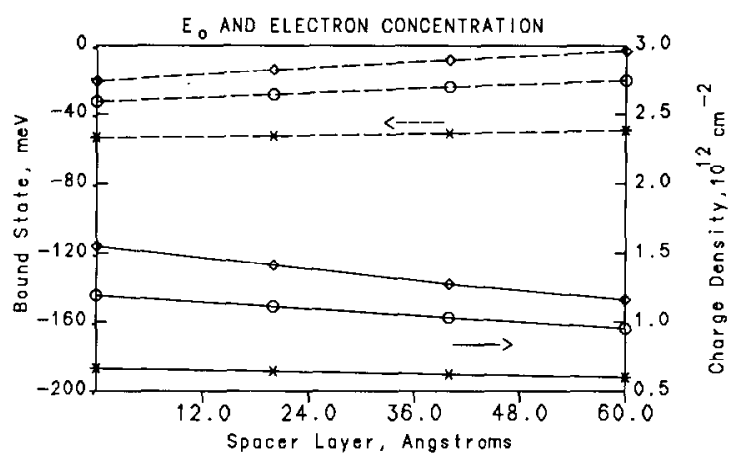

(b)

Fig. 1. Variation of the bound state position and bound state charge as a function of (a) well doping, (b) spacer layer thickness. The various curves represent different emitter and collector doping levels: $(\diamond) 1 \times 10^{18} \mathrm{~cm}^{-3},(0) 5 \times 10^{17} \mathrm{~cm}^{-3}$, and $(\times) 1 \times 10^{17} \mathrm{~cm}^{-3}$. For (a) $0 \AA$ spacer layers were used while the results in (b) assume a well doping of $1 \times 10^{17} \mathrm{~cm}^{-3}$.

barriers was $0.092 m_{0}$, and the temperature was $300 \mathrm{~K}$. These material parameters correspond to a GaAs/AlGaAs/ $/ \mathrm{In}_{0.20} \mathrm{Ga}_{0.80} \mathrm{As} / \mathrm{AlGaAs} / \mathrm{GaAs}$ RTS.

\section{Experimental results}

Some experimental results for various deep well RTS's have been presented before [4,5]. A GaAs based structure with a deep quantum well was grown by $\mathrm{MBE}$ and tested as a two terminal device. The device $I(V)$ at room temperature and $77 \mathrm{~K}$ along with the derivative of the current at room temperature is shown in fig. 2a. This device has barrier widths of $40 \AA$, well width of $60 \AA$, and spacer layers of $30 \AA$ on each side. The barriers are of AlAs. The deep well is obtained by using a $\operatorname{In}_{0.20} \mathrm{Ga}_{0.80}$ As well. Note that no negative differential resistance (NDR) exists at room temperature, however, there is a nonlinearity at about $110 \mathrm{mV}$. The $I(V)$ at $77 \mathrm{~K}$ shows a distinct NDR at $365 \mathrm{mV}$ but the nonlinearity at $110 \mathrm{mV}$ still persists. The value of the peak voltage agrees well with the expected first state resonance calculated from the above mentioned simulation. Single quantum well time resolved photoluminescence studies are under way to independently confirm the existence of charge in the bound state.

Fig. $2 b$ shows the $I(V)$ for a device where the well is made of $\mathrm{In}_{0.70} \mathrm{Ga}_{0.30}$ As and the emitter and collector are of InGaAs lattice matched to InP. In this device the the barriers are of InAlAs and are $30 \AA$ wide. The well width is $70 \AA$. Spacer layers of $50 \AA$ are used. The observed NDR is believed to be from the first excited state.

\section{Device applications}

Since the device $I(V)$ exhibits a NDR all the applications proposed for conventional RTS can also be implemented in this structure. However, for some device applications this structure presents advantages over the conventional RTS. For a high frequency source it has been shown that the performance is directly proportional to the peak current and device capacitance ralio [3]. By using the second quasi bound state for conduction a higher current density can be obtained. For a microwave detector on the other hand performance is related to the nonlinearity of the $I(V)$. Since the deep quantum well structures allow for the tailoring of the quasi bound state relative to the band edge, in theory a structure could be designed where a high nonlinearity in the $I(V)$ curve exists very close to the origin and thus the diode could be used as a detector without any external bias. It has also been shown that deep quantum well structures can have a better absorption coefficient than conventional RTS [6] for optical modulation.

A potentially very useful application of the structure could be as a charge transfer device. The 

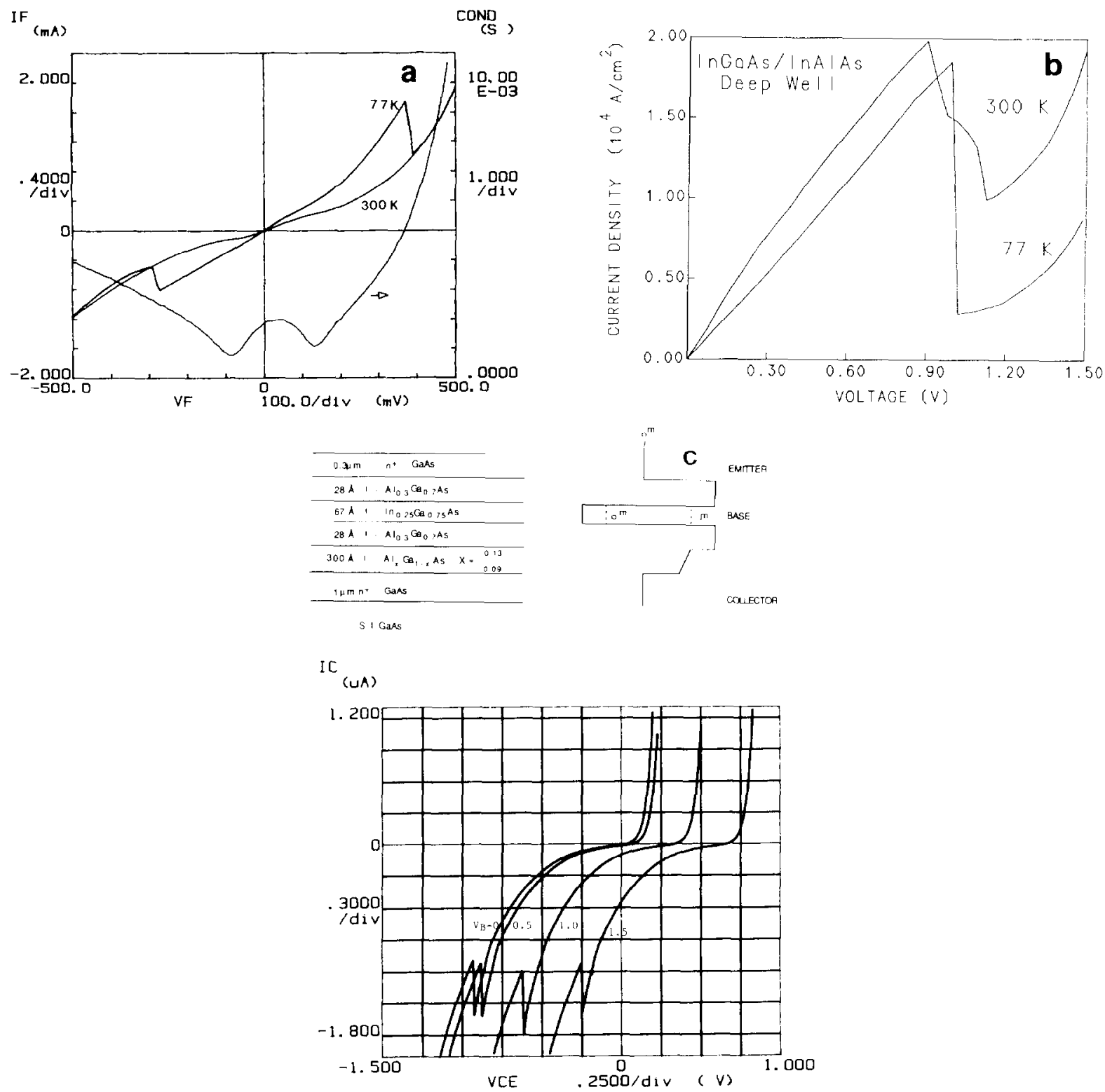

Fig. 2. (a) $I\left(l^{\prime}\right)$ at room temperature and $77 \mathrm{~K}$ for the GaAs deep-well structure. Also shown is the derivative of the current at room tomperature. (b) $I(V)$ for the InP deep-well structure. (c) Device structure and $I(V)$ for a deep-well structure as a three terminal device. The NDR region is modulated as a function of base voltage.

property is due to the simple fact that at zero bias there exists in the well a significant amount of charge and as bias is applied the charge "packet" is injected into the collector region. If this charge packet can be successfully sensed then the possibility exists of implementing charge transfer de- vices based on deep quantum well structures. Since the structure is a unipolar device charge retention will be easier compared to the present charge coupled device where charge recombination severely limits the charge retention time. Moreover, since the charge is transferred through single 
barrier tunneling the charge transfer time should be extremely fast.

Another important application of the deep well RTS is as a resonant tunneling transistor. Since there is charge in the well this structure is much better suited for a transistor than the conventional structure $[7,8]$. The structure proposed in ref. [8] has been fabricated and the preliminary results are shown in fig. 2c. By applying a voltage on the base the region of the NDR in the $I(V)$ plot is modulated. This, to the best of our knowledge, is the first observation of such an effect in a double barrier RTS. Similar results have previously been reported for a slightly different structure [9].

\section{Conclusion}

The deep quantum well RTS has been studied with a self-consistent simulation showing the unique properties of this structure such as the presence of charge in the quantum well at zero bias. Experimental results in the GaAs and InP based heterostructures have been presented and finally a number of device applications have been discussed. It is believed that by carefully understanding the operation of this structure electronic devices with useful functions can be fabricated.

\section{Acknowledgments}

We would like to thank J. Pamulapati, Dr. J. Oh, and Professor Bhattacharya for material growth. This work was supported by the ARO under the URI program contract no. DAAL03-87K-0007.

\section{References}

[1] R.K. Mains, J.P. Sun and G.I. Haddad, Appl. Phys. Lett. 24 (1989).

[2] W.R. Frensley, presented at the Int. Symp. on Nanostructure Physics and Fabrication, College Station, Texas, March 1989.

[3] I. Mehdi, R.K. Mains and G.I. Haddad, Micro. Opt. Tech. Lett. 2 (1989) 172.

[4] M.A. Reed and J.W. Lee, Appl. Phys. Lett. 50 (1987) 845.

[5] G.S. Lee. K.Y. Hsieh and R.M. Kolbas, Superlattices Microstruct. 4 (1988) 537.

[6] I. Mehdi, G.I. Haddad and R.K. Mains, Superlattices Microstruct. 5 (1989).

[7] G.I. Haddad, R.K. Mains, U.K. Reddy and J.R. East, Superlattices Microstruct. 5 (1989) 443.

[8] J.N. Schulman and M. Waldner, J. Appl. Phys. 63 (1988) 2859.

[9] F. Beltram, F. Capasso, S. Luryi, S.-N.G. Chu, A.Y. Cho and D.L. Sivco, Appl. Phys. Lett. 53 (1988) 219. 